\title{
Hyper-Solitons in Nematic Liquid Crystals
}

\author{
A. Strinic ${ }^{a, b, *}$, M. Petrović $\tilde{c}^{a, b}$ And M. Belićc ${ }^{b}$ \\ ${ }^{a}$ Institute of Physics, P.O. Box 57, 11001 Belgrade, Serbia \\ ${ }^{b}$ Texas A\&M University at Qatar, P.O. Box 23874 Doha, Qatar
}

\begin{abstract}
We study laser light propagation in a cell containing a liquid crystal in the nematic phase. We launch hyper-Gaussian beams and follow their behavior within the cell, in time and in three spatial dimensions, utilizing an appropriately developed theoretical model and a numerical procedure based on the fast Fourier transform. We demonstrate the formation of stable "hyper-soliton" breathers in a narrow region of beam intensities, for fixed other parameters. Hyper-solitons are similar in appearance and behavior to the usual solitons, formed by launching the usual Gaussian beams; however noticeable differences persist.
\end{abstract}

PACS numbers: 42.70.Df, 42.65.Tg

\section{Introduction}

Propagation of self-focused beams [1] in nematic liquid crystals (NLCs) [2] has been the subject of significant interest in recent years [3-9]. NLCs are known to exhibit enormous optical nonlinearities, due to their large refractive index anisotropy, coupled with the optically-induced collective molecular reorientation. We investigate the propagation of single self-trapped laser beams in NLCs. Starting from the standard equations describing the nonlocal and nonlinear interaction of light and NLC, we develop a numerical procedure that treats these equations in three spatial dimensions and time. We launch hyperGaussian beams of order 4, and compare the behavior of the resulting hyper-solitons with the solitons formed by launching ordinary Gaussian beams. We vary only a few input beam parameters: the input intensity and the full width at half maximum (FWHM) of the beam, and demonstrate the formation of soliton breathers in a narrow region of beam intensities. We present the optical field intensity $I(0,0, z)$ along the propagation axis, in the middle of the crystal and breathing soliton transverse profiles at the cell exit $(x, y)$ plane.

\section{The model}

In the presence of an externally applied (low frequency) voltage the spatial evolution of a slowly-varying beam envelope $A$, linearly polarized along the $x$ axis and propagating along the $z$ axis, the dimensionless paraxial wave equation [3]:

$$
2 \mathrm{i} \frac{\partial A}{\partial z}+\Delta_{x, y} A+\alpha\left(\sin ^{2} \theta-\sin ^{2} \theta_{0}\right) A=0,
$$

where $\Delta_{x, y}$ is the transverse Laplacian. The temporal evolution of the angle of reorientation $\theta$ is given by the dimensionless diffusion equation $[7,10,11]$ :

\footnotetext{
* corresponding author; e-mail: strinic@phy.bg.ac.yu
}

$$
\frac{2 \gamma x_{0}^{2}}{K \tau} \frac{\partial \theta}{\partial t}=2 \Delta_{x, y} \theta+\left(\beta+\alpha|A|^{2}\right) \sin (2 \theta) .
$$

Hard boundary conditions on the molecular orientation at the transverse borders of the liquid-crystal cell are imposed

$$
\theta(x=-D / 2)=\theta(x=D / 2)=2^{\circ} .
$$

Here, $\alpha=k_{0}^{2} x_{0}^{2} \Delta \varepsilon^{\mathrm{OPT}}, \beta=\left(\varepsilon_{0} x_{0}^{2} \Delta \varepsilon^{\mathrm{DC}}\left|E^{\mathrm{DC}}\right|^{2}\right) / K, \gamma$ is the viscous coefficient, $K$ is Frank's elastic constant, $\tau$ is the director relaxation time, $k=k_{0} n_{0}$ is the wave vector in the medium, $x_{0}$ the transverse scaling length, $\Delta \varepsilon^{\mathrm{OPT}}$ is the optical permittivity anisotropy of the liquid-crystal molecules, $\Delta \varepsilon^{\mathrm{DC}}$ is the static permittivity anisotropy of the liquid-crystal molecules and $E^{\mathrm{DC}}$ is the applied field strength. $\theta$ is the overall tilt angle (the total orientation of the molecules with respect to the $z$ axis), owing to both light and voltage: $\theta=\theta_{0}+\hat{\theta}$, where the angle $\theta_{0}$ accounts for the molecular orientation induced by the static electric field only, while the quantity $\hat{\theta}$ corresponds to the optically induced molecular reorientation.

By solving Eqs. (1) and (2) we describe the beam propagation in three spatial dimensions and time. We developed a novel numerical procedure, based on fast Fourier transform (FFT), utilizing our prior experience in treating the beam propagation in NLC $[5,12]$. The initial distribution $\theta_{0}$ is determined in the beginning, independent of the solution of the full system of Eqs. (1) and (2), using boundary conditions and a successive over-relaxation (SOR) algorithm for solving partial differential equations. This approach gives us a more realistic physical modeling of the system [13, 14]. We present here only the results when steady state is reached.

\section{Results of numerical simulation}

We investigate the behavior of propagating hyper-Gaussian beams in a bulk NLC. The effect of the input intensity variation on the single hyper-Gaussian beam propagation is presented in Fig. 1. We show the steadystate beam intensities $I(0, y, z)$ and the angle reorientation distributions $\hat{\theta}(0, y, z)$, as functions of the propagation distance, for different input beam intensities. For 
smaller intensities self-focusing is too weak to keep the beam tightly focused, so initially it spreads and then slightly refocuses (Fig. 1a and $1 \mathrm{~b}$ ). By increasing the beam intensity (Fig. 1c) a threshold for stable solitonic propagation is achieved, in the form of hyper-soliton breathers: the beams preserve their new shape, but their characteristic width and maximum intensity breathe periodically as they propagate. For still higher intensities (Fig. 1d) irregular behavior of the beam is observed. This behavior is very similar to the behavior of regular Gaussian solitons, as described in $[13,14]$.
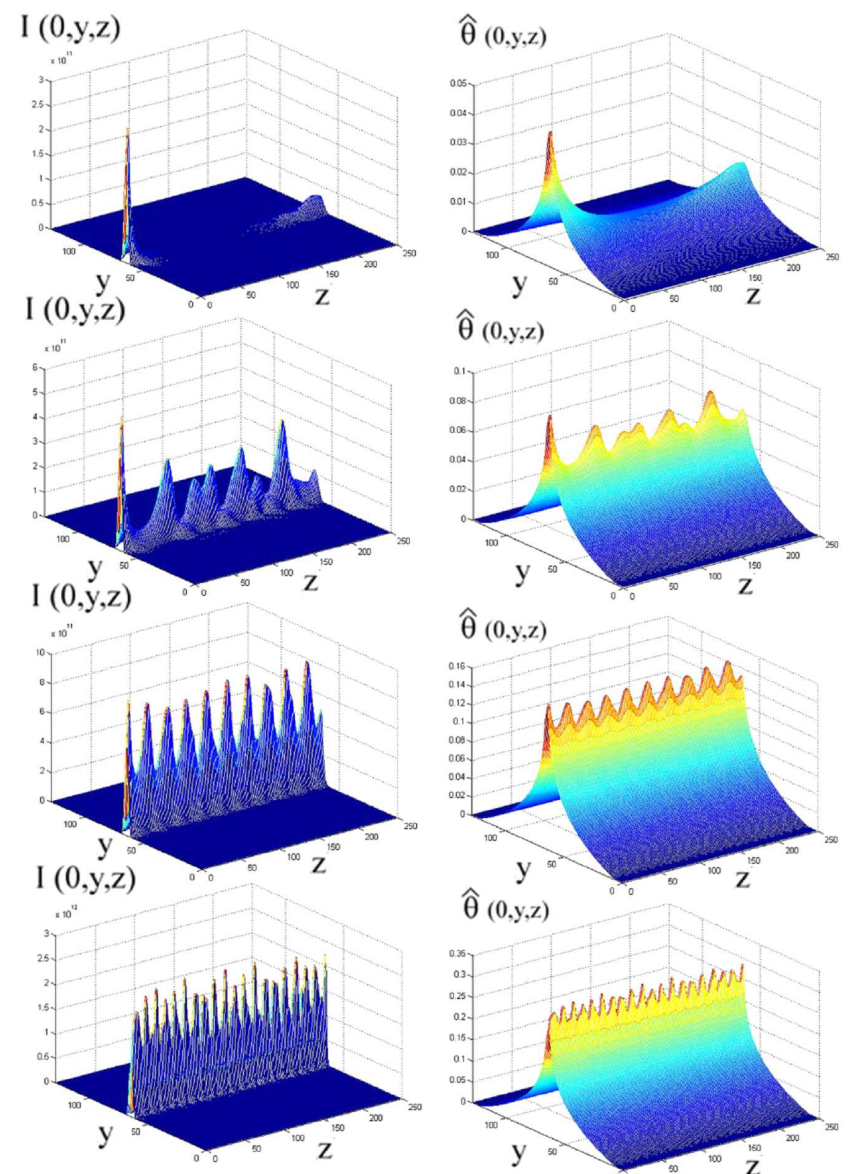

Fig. 1. Development of hyper-soliton breathers. Beam propagation along the $z$-direction is depicted for intensities $I(0, y, z)$ in the middle of the crystal (the first column) and reorientation angles $\hat{\theta}(0, y, z)$ (the second column). Input intensities: the first row $I=$ $2 \times 10^{+11} \mathrm{~V}^{2} / \mathrm{m}^{2}$, the second row $I=3.8 \times 10^{+11} \mathrm{~V}^{2} / \mathrm{m}^{2}$, the third row $I=6 \times 10^{+11} \mathrm{~V}^{2} / \mathrm{m}^{2}$, and the fourth row $I=1 \times 10^{+12} \mathrm{~V}^{2} / \mathrm{m}^{2}$. Parameters: $\Delta \varepsilon^{\mathrm{OPT}}=0.4$, $\Delta \varepsilon^{\mathrm{DC}}=14.5, \mathrm{FWHM}=2.5 \mu \mathrm{m}$ and $L=1.5 \mathrm{~mm}$.

When the same sequence of increasing input intensity simulations is repeated for different input widths (FWHM), for fixed parameters $\Delta \varepsilon^{\mathrm{OPT}}=0.4$ and $\Delta \varepsilon^{\mathrm{DC}}=14.5$, a similar behavior is found. For each input beam width, appropriate input beam intensity can be found, needed to establish the existence of a hyper- soliton breather (Fig. 2a). In Fig. 2a we show the cases of the hyper-soliton breather propagation, for FWHM = $2.5 \mu \mathrm{m}, 3 \mu \mathrm{m}$, and $3.5 \mu \mathrm{m}$. For smaller FWHM higher input intensities are needed for the emergence of breathing solitons. We also note that the changes in FWHM causes the changes in the period of oscillation.

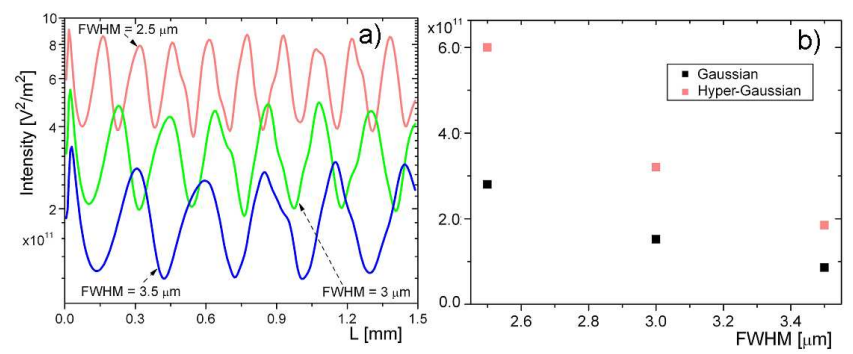

Fig. 2. (a) Stable hyper-soliton breather propagation in the $z$ direction: the input beam intensity in the middle of the crystal is shown for different input FWHM widths. For each input beam width appropriate input beam intensity is found for the existence of a breathing soliton. (b) Input intensities of breathing solitons versus input FWHM widths, for Gaussian and hyperGaussian inputs. Parameters: $\Delta \varepsilon^{\mathrm{OPT}}=0.4, \Delta \varepsilon^{\mathrm{DC}}=$ 14.5 and $L=1.5 \mathrm{~mm}$.
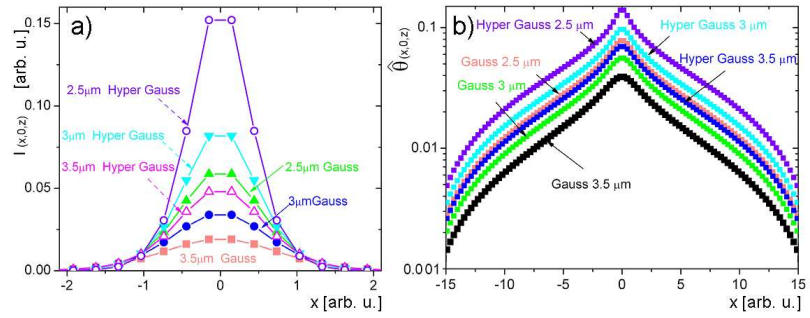

Fig. 3. Soliton breathers transverse profiles in the narrow region of stability. Beam intensity (a) and optically induced molecular reorientation (b), for Gaussian and hyper-Gaussian beams, for different input FWHM. Note the logarithmic scale for the reorientation angle profiles. Parameters: $\Delta \varepsilon^{\mathrm{OPT}}=0.4, \Delta \varepsilon^{\mathrm{DC}}=14.5$ and $L=1.5 \mathrm{~mm}$.

In Fig. 2b we compare the formation of Gaussian and hyper-Gaussian breathers. We display the input intensities of breathing solitons as functions of the input widths (FWHM ranging from $2.5 \mu \mathrm{m}$ to $3.5 \mu \mathrm{m}$ ), for Gaussian and for hyper-Gaussian beams, in the soliton formation region. It is seen that for the hyper-Gaussian beams, higher input intensities are needed for the formation of hyper-soliton breathers.

In Fig. 3 we show the transverse profiles of solitons at the exit $(x, y)$ plane: the intensity and the optically induced molecular reorientation, for Gaussian and hyper-Gaussian beams. It is evident that the shapes are very similar; although it appears that the hyper-solitons are taller than the usual solitons for the same widths. This comes about because higher input intensities of the 


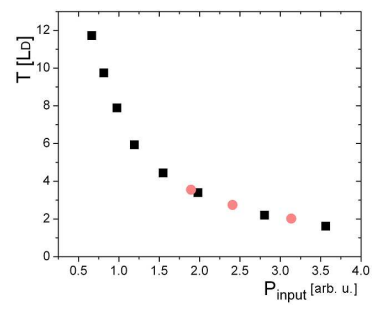

Fig. 4. Period of soliton breathers versus input power, for Gaussian (black symbols) and hyper-Gaussian beams (grey symbols), for different input FWHM. Parameters as in Fig. 3.

hyper-Gaussian beams are needed for the formation of hyper-solitons. The same applies to the reorientation angle profiles. A comparison between the beam intensity and the reorientation angle distributions shows that the reorientation angle distribution is always much wider, owing to strong nonlocality for the given parameters, $[4,11,15,16]$.

In Fig. 4 we show the period the period of soliton breathers of the breathing solitons as functions of the input power, for Gaussian and hyper-Gaussian beams. One can see that the period decreases as the input power increases. It appears that the periods for Gaussian and hyper-Gaussian beams follow the same inverse-power law, as functions of the input power.

\section{Conclusion}

In this paper we investigated the propagation of hyperGaussian laser beams in a NLC. We displayed the behavior of input hyper-Gaussian beams in time and three spatial dimensions. We demonstrated the formation of hyper-soliton breathers and examined the differences from the usual soliton breathers in NLCs. In particular, we examined the influence of input intensities and input FWHM on the formation of soliton breathers. We found that the typical stable soliton propagation mode is breathing, be it for the Gaussian or for the hyperGaussian input beams. We determined the period of breathing solitons and found that the period follows an inverse power law as a function of the input intensity

\section{Acknowledgments}

This study is supported by the Qatar National Research Foundation project NPRP 25-6-7-2 and by the
Ministry of Science and Technological Development of the Republic of Serbia, under the project OI 141031.

\section{References}

[1] Y.S. Kivshar, G.P. Agrawal, Optical Solitons, Academic Press, San Diego 2003.

[2] I.C. Khoo, Liquid Crystals: Physical Properties and Nonlinear Optical Phenomena Wiley, New York 1995.

[3] M. Peccianti, C. Conti, G. Assanto, A. De Luca, C. Umeton, J. Nonlin. Opt. Phys. Mater. 12, 525 (2003).

[4] J.F. Henninot, M. Debailleul, M. Warenghem, Mol. Cryst. Liq. Cryst. 375, 631 (2002).

[5] G. Assanto, M. Peccianti, K.A. Brzdąkiewicz, J. Nonlin. Opt. Phys. Mater. 12, 123 (2003).

[6] G. D'Alessandro, A.A. Wheeler, Phys. Rev. A 67, 023816 (2003).

[7] X. Hutsebaut, C. Cambournac, M. Haelterman, J. Beeckman, K. Neyts, J. Opt. Soc. Am. B 22, 1424 (2005).

[8] A.I. Strinić, D.V. Timotijević, D. Arsenović, M.S. Petrović, M.R. Belic, Opt. Express 13, 493 (2005).

[9] P.D. Rasmussen, O. Bang, W. Krolikowski, Phys. Rev. E 72, 066611 (2005)

[10] J. Beeckman, K. Neyts, X. Hutsebaut, C. Cambournac, M. Haelterman, Opt. Express 12, 1011 (2004).

[11] J. Beeckman, K. Neyts, X. Hutsebaut, C. Cambournac, M. Haelterman, IEEE J. Quantum Electron. 41, 735 (2005).

[12] A. Strinić, D. Jović, M. Petrović, D. Timotijević, N. Aleksić, M. Belić, Opt. Express 14, 12310 (2006).

[13] A.I. Strinic, M.R. Belic, Acta Phys. Pol. A 112, 877 (2007).

[14] A. Strinić, M. Petrović, D. Timotijević, N. Aleksić, M. Belić, Opt. Express 17, 11698 (2009).

[15] M. Peccianti, C. Conti, G. Assanto, , A. De Luca, C. Umeton, J. Nonlin. Opt. Phys. Mater. 12, 525 (2003).

[16] J.F. Henninot, J.F. Blach, M. Warenghem, J. Opt. A, Pure Appl. Opt. 10, 085704 (2008). 